\title{
Philosophiques
}

\section{Quelques énigmes concernant le contrôle de soi}

\section{Michael Smith}

Volume 27, numéro 2, automne 2000

URI : https://id.erudit.org/iderudit/004985ar

DOI : https://doi.org/10.7202/004985ar

Aller au sommaire du numéro

Éditeur(s)

Société de philosophie du Québec

ISSN

0316-2923 (imprimé)

1492-1391 (numérique)

Découvrir la revue

Citer cet article

Smith, M. (2000). Quelques énigmes concernant le contrôle de soi. Philosophiques, 27(2), 287-304. https://doi.org/10.7202/004985ar

\section{Résumé de l'article}

L'idée selon laquelle un agent jouissant du contrôle de soi peut tantôt exercer ce contrôle, et tantôt faillir à l'exercer occupe une place centrale dans notre compréhension de la resposabilité morale. Mais lorsqu'on tente de préciser et d'expliciter davantage la notion de contrôle de soi, on semble se heurter à la contradiction et au paradoxe. Mon but dans cet article est de formuler et de résoudre les diverses énigmes soulevées par cette notion.
Ce document est protégé par la loi sur le droit d'auteur. L'utilisation des services d’Érudit (y compris la reproduction) est assujettie à sa politique d'utilisation que vous pouvez consulter en ligne.

https://apropos.erudit.org/fr/usagers/politique-dutilisation/ 


\title{
Quelques énigmes concernant le contrôle de soi
}

\author{
MICHAEL SMITH \\ Australian National University \\ Msmith@coombs.anu.edu.au
}

RÉSUMÉ. - L'idée selon laquelle un agent jouissant du contrôle de soi peut tantôt exercer ce contrôle, et tantôt faillir à l'exercer occupe une place centrale dans notre compréhension de la resposabilité morale. Mais lorsqu'on tente de préciser et d'expliciter davantage la notion de contrôle de soi, on semble se heurter à la contradiction et au paradoxe. Mon but dans cet article est de formuler et de résoudre les diverses énigmes soulevées par cette notion.

ABSTRACT. - The idea that agents who possess self-control may sometimes exercise their control while at other times failing to do so is central to our understanding of moral responsibility. When we try to make the idea of self-control more explicit and precise, however, it appears to be riddled with contradiction and paradox. My aim in this paper is to formulate and solve the various puzzles about self-control that arise.

Chaque jour, en allant au travail, Jean s'arrête au supermarché afin d'acheter sa ration quotidienne de tablettes de chocolat. Il sait très bien qu'il ne devrait pas manger autant de chocolat. Étant donné qu'il a dépassé la quarantaine et qu'il ne fait aucun exercice physique, le chocolat qu'il consomme ne fait qu'aggraver un problème de poids déjà préoccupant. Bien qu'il croit qu'en mangeant autant de chocolat il engraissera et augmentera les risques d'avoir un problème cardiaque, cela n'y change rien. On l'entend souvent dire : « Je sais que je ne devrais pas manger autant de chocolat. Je sais que je devrais me contrôler. Mais j'en suis incapable. J'aime tellement çà. »

Je n'ai nul doute que l'histoire de Jean vous est trop bien connue. Votre vice n'est peut-être pas le chocolat. C'est peut-être la cigarette, l'alcool ou la drogue ; c'est peut-être les autos, les motos, les livres, la télévision, le travail ou l'exercice physique. Il ne semble pas y avoir de limites aux choses que nous, humains, nous permettons, alors que nous ne le devrions pas. Mais peu importe le vice particulier de chacun, l'important est que nous savons tous ce qu'est penser que nous sommes en train de faire quelque chose que nous ne devrions pas faire, et nous savons tous ce que c'est que de se rendre compte que c'est le moment de se contrôler.

Jean dit qu'il ne peut se contrôler, et peut-être que ce qu'il dit est vrai. Si c'est vrai, alors, bien sûr, il pourrait ne pas être avisé de notre part de le tenir pour responsable de ses actions. S'il ne peut réfréner son désir de chocolat de son propre chef, alors peut-être a-t-il besoin que l'un d'entre nous l'emmène chez les Chocoholiques Anonymes. Mais, dans un cas comme celui de Jean, c'est au moins une question discutable que celle de savoir si ce qu'il 
dit est vrai. Si ce qu'il dit est faux — s'il ment, ou s'il se trompe lui-même, et peut se contrôler - alors, en l'absence d'autres considérations qui pourraient suggérer le contraire, il est légitime pour nous de le tenir pour responsable de ce qu'il fait. C'est du moins ce que nous dit le bon sens. Dans le présent texte, je veux examiner de plus près la question de savoir si le bon sens a du sens.

Ma stratégie, dans les pages qui suivent, sera d'examiner certains détails de l'histoire de Jean. Je veux utiliser ces derniers afin de soulever un certain nombre d'énigmes concernant le contrôle de soi. Ces énigmes se présentent à nous parce que, comme nous le verrons, lorsque nous essayons de rendre plus explicite et plus précise l'idée de contrôle de soi, celle-ci semble pleine de contradictions et de paradoxes. Bien que mon but soit de résoudre ces énigmes, je dois dire dès le départ que ma solution requiert certaines assomptions que d'aucuns ne voudront pas accepter. De mon point de vue, si vous ne voulez pas les accepter, alors votre seul choix est d'abandonner l'idée que nous avons parfois besoin de nous contrôler et que nous sommes capables de le faire. Étant donnée la relation intime entre cette idée du sens commun et la possibilité d'assignation de responsabilité morale, je ne veux pas abandonner cette idée. Je préfère accepter les thèses controversées. Mais c'est une question à laquelle vous devez apporter vous-même une réponse.

\section{Comment peut-on, en toute connaissance de cause, agir d'une certaine façon alors que, croyons-nous, nous ne devrions pas agir de cette façon?}

\section{Selon Thomas Hobbes :}

(...) l'objet, quel qu'il soit, de l'appétit ou du désir d'un homme, est ce que pour sa part celui-ci appelle bon; et il appelle manvais l'objet de sa haine et de son aversion; sans valeur et négligeable, l'objet de son dédain. En effet, ces mots de bon, de mauvais et de digne de dédain s'entendent toujours par rapport à la personne qui les emploie; car il n'existe rien qui soit tel, simplement et absolument; ni aucune règle commune du bon et du mauvais qui puisse être emprunté à la nature des objets eux-mêmes (...) Hobbes, 1651, première partie, chapitre 6, p. $48^{1}$

La position défendue ici par Hobbes est très répandue de nos jours.

Cette thèse veut que, à cause de cette étroite relation entre ce que nous appelons le bien et le mal, d'une part, et ce que nous faisons, d'autre part, nos désirs et nos aversions, qui sont la cause de ce que nous faisons doivent donner un contenu aux faits que nous croyons être bien et mal. La raison pour laquelle les mots «bien » et «mal » sont utilisés, comme le mentionne

1. Hobbes, T. Leviathan, Introduction par C.B. McPherson, Harmmondsworth, Penguin, 1968. Trad. Fr. par F. Tricaud, Léviathan, Paris, Sirey, 1971. 
Hobbes, «par rapport à la personne qui les emploie » est donc parce qu'il n'y a aucun fait non relationnel. - aucun fait sauf le fait que la chose est désirée - auquel une croyance puisse se raccrocher d'une manière requise pour l'action.

Si Hobbes a raison à propos de la signification de «bien » et de " mal », cependant, il semblerait que Jean soit dans l'erreur lorsqu'il dit qu'il ne devrait pas manger autant de chocolat. Après tout, si nous traduisons ce que Jean affirme dans les termes de Hobbes, il dit qu'il sait que c'est mal de manger autant de chocolat. Ceci signifie simplement qu'il éprouve de l'aversion à manger autant de chocolat, et désire ne pas en manger autant. Mais, étant donnée la manière dont nous avons raconté l'histoire de Jean, ce dernier n'éprouve pas d'aversion à manger autant de chocolat. En fait, il a un très fort désir de manger du chocolat! Si nous le voulons, nous pouvons même embellir notre récit et imaginer que Jean n'a aucun désir de ne pas manger de chocolat. Mais, même si nous ajoutons ce détail, il semble que nous pouvons toujours l'imaginer dire, sincèrement et avec vérité, que bien qu'il ait un fort désir de manger du chocolat, et pas de désir de ne pas le faire, il sait néanmoins qu'il ne devrait pas manger autant de chocolat. Mais comment peut-il en être ainsi ? Si Hobbes a raison, Jean se contredirait, tout simplement.

Nous avons donc la première énigme du contrôle de soi. Il faut rendre compte de ce que "doit" signifie lorsque, dans les cas où nous perdons le contrôle de nous-mêmes, nous disons savoir que nous faisons une chose que nous ne devrions pas faire. Mais, les analyses les plus évidentes sur le marché - les variations sur l'analyse de Hobbes, par exemple — non seulement n'apporteront pas de réponse, mais elles semblent impliquer une contradiction dans ce que nous disons. J'aimerais soutenir que la solution à cette énigme réside non pas dans l'abandon complet de la position de Hobbes, mais dans une modification de cette dernière. Cette modification nous vient non pas de la Renaissance mais des Lumières. Afin de présenter la modification que j'ai à l'esprit, j'aimerais commencer par décrire le cas de Jean de façon un peu plus détaillée.

Supposons que Jean ait deux désirs intrinsèques : un désir d'être en bonne santé et un désir, plus faible, d'éprouver du plaisir. Étant donné que Jean a aussi de nombreuses croyances concernant la relation moyen/fin, il s'ensuit que s'il était pleinement intrumentalement rationnel, il aurait aussi des désirs extrinsèques : il aurait d'autres désirs pour ce qu'il ne désire pas intrinsèquement, mais simplement comme moyens pour atteindre ce qu'il désire intrinsèquement. Supposons donc que Jean, parce qu'il désire intrinsèquement le plaisir et croit que manger du chocolat donne du plaisir, s'il était pleinement instrumentalement rationnel, extrinsèquement désirerait manger du chocolat; supposons aussi que, parce qu'il désire intrinsèquement être en bonne santé et croit que manger du chocolat fera qu'il ne sera pas en bonne santé, il désirerait aussi, s'il était pleinement instrumentalement 
rationnel, ne pas manger de chocolat. Avec cet arrière-fond à l'esprit, demandons-nous ce que Jean devrait faire.

Une interprétation assez naturelle de cette question s'offre d'elle-même. Dans cette interprétation, ce que nous voulons savoir est ce que Jean voudrait le plus faire, dans les circonstances, s'il était pleinement instrumentalement rationnel. Lorsque nous posons la question dans ces termes, la réponse paraît relativement claire. Ce que Jean devrait faire - c'est-à-dire ce qu'il devrait vouloir faire, dans les circonstances, s'il était pleinement instrumentalement rationnel - est de s'empêcher de manger du chocolat. Il devrait s'empêcher de manger du chocolat, parce que son désir intrinsèque d'être en bonne santé est plus fort que son désir intrinsèque d'éprouver du plaisir. Donc, s'il était pleinement instrumentalement rationnel, les forces de ses désirs extrinsèques de manger et de ne pas manger de chocolat suivraient leurs cours.

Bien sûr, même si c'est ce que Jean devrait faire, il ne s'en suit pas que c'est ce qu'il fera. Ce qu'un agent fera est fonction du désir intrinsèque qu'il a de fait, et pas de celui qu'il aurait s'il était pleinement instrumentalement rationnel. Le désir d'être en bonne santé de Jean peut être sans impact sur son comportement, sans d'abord se combiner à ses croyances concernant la relation moyen/fin afin d'engendrer le désir extrinsèque de faire ce qu'il croit être un moyen d'être en bonne santé. Donc, même s'il peut être vrai que le désir de Jean de ne pas manger de chocolat devrait être plus fort que son désir d'en manger - en ce sens qu'il serait plus fort, s'il était pleinement instrumentalement rationnel - il peut toujours arriver qu'il ne soit pas le plus fort de fait parce que Jean peut ne pas être pleinement instrumentalement rationnel.

En conséquence de quoi, il me semble que nous avons trouvé une interprétation assez naturelle de ce que Jean signifie lorsqu'il dit « Je sais que je ne devrais pas manger autant de chocolat ". Si la structure des désirs de Jean est telle que décrite plus haut, alors la thèse veut qu'il exprime sa croyance qu'il désirerait extrinsèquement ne pas manger de chocolat, dans les circonstances, s'il était pleinement instrumentalement rationnel. Cependant, puisqu'il n'est pas pleinement intrumentalement rationnel, dans les circonstances, il n'a pas en fait un tel désir extrinsèque. Puisque ce qu'il fait est fonction des désirs extrinsèques qu'il a en fait, il s'ensuit que si le seul désir intrinsèque de Jean qui réussit à engendrer un désir pour les moyen est un désir de plaisir, alors il aura, et agira de fait, sur la base d'un désir extrinsèque de manger du chocolat. Je répète : ce peut ne pas être le désir extrinsèque le plus fort qu'il aurait s'il était instrumentalement irrationnel, mais ce peut être le plus fort désir extrinsèque qu'il a malgré tout.

Cette interprétation de ce que dit Jean me semble aussi naturelle. Après tout, les échecs de la rationalité instrumentale, peut-être inhabituels, ne sont pas inusités au point d'être incompréhensibles. Nous savons tous ce que c'est, lorsque sentir et goûter du chocolat rend particulièrement saillant et aigu le plaisir d'en manger, au moins lorsque nous le comparons à la nature 
inerte et terne de notre savoir que s'empêcher d'en manger améliorera notre état de santé. Dans de telles situations, nous nous dirions, tout comme Jean, que nous devrions nous contrôler. Nous devrions nous contrôler parce que de cette manière seulement nos actions servent-elles nos désirs, étant donné nos croyances quant à la manière dont elles devraient le faire.

L'énigme initiale, concernant le contrôle de soi, était engendrée en adoptant la position de Hobbes selon laquelle ce que chacun de nous, pour sa part, appelle «bien » et «mal » est affaire des désirs et des aversion que nous avons de fait. La solution à cette énigme réside dans l'identification d'une faiblesse dans la position de Hobbes, une faiblesse qui nous oblige à la modifier. Lorsque nos désirs et nos aversions sont le produit d'une irrationalité instrumentale, il est simplement peu plausible de supposer qu'ils représentent ce que nous considérons comme bien et mal. C'est peu plausible, parce que serions rapidement d'accord pour dire que ce sont des désirs et des aversions que nous ne devrions pas avoir, en un sens légitime de ce terme, c'est-à-dire en ce sens que nous ne les aurions pas si nous étions pleinement instrumentalement rationnels.

Ce qui semble toujours plausible, néanmoins, est une version modifiée de la position de Hobbes, une version modifiée dans l'esprit des Lumières et due à Kant, selon laquelle nos désirs et nos aversions doivent eux-mêmes se former en accord avec des principes rationnels. Ce que chacun d'entre nous, pour sa part, appelle «bien » et « mal » est toujours affaire de la relation que les choses entretiennent avec nous. Mais cette relation n'est pas celle d'être une chose que nous désirons de fait ou pour laquelle nous avons une aversion de fait. C'est plutôt celle d'être une chose que nous désirerions, ou pour laquelle nous éprouverions de l'aversion, si nous étions dans un état plus pleinement rationnel, c'est-à-dire si nous avions des désirs et des aversions qui furent formées en accord avec les principes rationnels qui les gouvernent, des principes rationnels comme la relation moyen/fin.

Une fois ainsi modifiée la position de Hobbes, cependant, il devient clair que l'irrationalité instrumentale peut n'être qu'une cause parmi d'autres du besoin de se contrôler. N'importe quelle critique rationnelle des désirs que nous avons de fait amènera un besoin correspondant d'exercer le contrôle de soi, c'est-à-dire un cas dans lequel nous désirons faire quelque chose alors que nous ne le devrions pas. La question que nous devons nous poser est donc celle de savoir s'il y a d'autres formes de critique rationnelle. Et la réponse est oui.

Imaginons une variation sur le cas décrit plus haut. Jean n'a qu'un désir intrinsèque : le désir de plaisir. Et il croit que manger du chocolat est une cause de plaisir. Bien sûr, il croit que c'est aussi une cause de mauvaise santé, et il croit que la mauvaise santé est déplaisante. Mais supposons que Jean croit que, tout compte fait, une vie en mauvaise santé au cours de laquelle il mange du chocolat est plus agréable qu'une vie en bonne santé au cours de laquelle il n'en mange pas. Si, dans ce genre de cas, nous pouvons toujours 
imaginer Jean dire, avec véracité, "Je sais que je ne devrais pas manger autant de chocolat ", alors la raison pour laquelle nous pouvons l'imaginer dire une telle chose ne peut être que nous supposons qu'il ne voudrait pas manger du chocolat s'il était pleinement instrumentalement rationnel parce que, par hypothèse, nous supposons que Jean désirerait manger du chocolat s'il était pleinement instrumentalement rationnel. La question que nous devons nous poser est donc celle de savoir si nous pouvons toujours imaginer Jean dire "Je sais que je ne devrais pas manger autant de chocolat».

Lorsque Jean dit qu'il ne devrait pas manger autant de chocolat, entendons-nous pour affirmer qu'il dit qu'il ne voudrait pas manger autant de chocolat, dans les circonstances, s'il était pleinement rationnel simpliciter, et entendons-nous aussi pour affirmer que la rationalité instrumentale est au moins un aspect de la pleine rationalité simpliciter. Y a-t-il d'autres aspects ? Il me semble qu'il y en a certainement. Un autre aspect est d'avoir des désirs qui soient libres de tout effet distordant, comme la dépression ; un autre est d'avoir des désirs qui soient éclairés ; un autre est d'avoir des désirs qui, réunis, forment un ensemble unifié et cohérent. On pourrait continuer ainsi pendant longtemps.

Ces autres exigences sur la rationalité simpliciter suggèrent que d'autres histoires sont possibles afin de rendre compte de la raison pour laquelle Jean n'a qu'un seul désir intrinsèque, un désir de plaisir, des histoires qui, chaque fois, condamneraient la structure désidérative comme un échec de la pleine rationalité. Ainsi, une première histoire donne comme raison pour laquelle Jean n'a qu'un désir intrinsèque, un désir de plaisir, son état dépressif et le fait que sa dépression a détruit ses autres désirs. Une seconde veut que Jean ait un seul désir intrinsèque, un désir de plaisir, et que son incapacité d'avoir d'autres désirs est en quelque sorte un produit de son ignorance. Une troisième veut que la raison pour laquelle Jean a un seul désir intrinsèque, un désir de plaisir, est qu'il a un ensemble de désirs moins que parfaitement cohérent. Je vais expliciter ces histoires avec un peu plus de détails, ne serait-ce que pour rendre claire l'idée que j'ai à l'esprit. Mettons l'accent sur l'exigence que l'ensemble des désirs soit pleinement éclairé.

Supposons que, bien que Jean n'ait pas de fait un désir intrinsèque d'être en bonne santé, il avait ce désir par le passé. Il a d'abord acquis ce désir comme sous-produit d'un programme de conditionnement physique dans lequel il s'était inscrit par curiosité. Mais, à mesure qu'il améliorait sa santé et gagnait un sens très aigu de ce qui constitue la bonne santé - l'énergie que l'on a, l'image du corps, l'ouverture de nouvelles possibilités, l'esprit clair et ainsi de suite - il a découvert qu'il voulait, intrinsèquement, vraiment être en bonne santé. En fait, c'était son plus fort désir intrinsèque, à tel point qu'il devint un fanatique du conditionnement physique. Cependant, supposons aussi que, pour une raison ou pour une autre, il ait été incapable de poursuivre son programme de conditionnement physique pendant un mois et que, lorsque ce mois se fut écoulé et qu'il s'est trouvé finalement capable d'y reve- 
nir, il s'est apercu qu'il ne voulait pas y revenir. Il n'avait plus un sens aigu de ce qui constitue une bonne santé - il avait plus ou moins oublié — et se rendit compte que, sans une constante réflexion sur ce qui constitue la bonne santé, son désir d'avoir une bonne santé avait tout simplement disparu.

Sur cet arrière-fond, demandons-nous ce à quoi a affaire Jean dans les circonstances. Si on interprète cela comme une demande quant à ce qu'il voudrait faire, dans les circonstances, s'il était pleinement rationnel — c'est-àdire, abstraction faite maintenant des autres exigences, s'il connaissait tous les faits pertinents et était pleinement instrumentalement rationnel - alors la réponse impliquerait, me semble-t-il, un conflit, un conflit déjà résolu.

D'une part, même s'il était pleinement éclairé, il aurait toujours ce désir intrinsèque de plaisir. S'il était, en plus, pleinement instrumentalement rationnel, alors il aurait aussi un désir intrinsèque de manger du chocolat pour avoir du plaisir. Une chose qu'il devrait donc faire, dans la mesure où ce sont là tous les faits, est de manger du chocolat. D'autre part, il y a aussi le désir d'être en bonne santé qu'il aurait encore s'il avait toujours un sens aigu de ce qui constitue une bonne santé — c'est-à-dire s'il était pleinement éclairé - et le désir intrinsèque de ne pas manger de chocolat comme moyen d'être en bonne santé qu'il aurait encore s'il était pleinement instrumentalement rationnel. Une autre chose qu'il devrait faire, donc, est de s'empêcher de manger du chocolat.

Jusqu'à maintenant, il y a conflit. Jean, à la lumière d'un ensemble de considérations, devrait manger du chocolat et, à la lumière d'autres considérations, devrait s'empêcher de manger du chocolat. Mais ce conflit est aisément résolu. Parce que par hypothèse, puisque si Jean était pleinement rationnel, alors son désir intrinsèque d'être en bonne santé serait plus fort que son désir intrinsèque de plaisir. Il s'ensuit que, s'il était pleinement rationnel, son désir extrinsèque de s'empêcher de manger du chocolat, sachant que manger du chocolat le rendra en mauvaise santé, serait plus fort que son désir extrinsèque de manger du chocolat, sachant que manger du chocolat est un moyen d'avoir du plaisir. Lorsque ces deux affirmations contenant « devrait " sont réunies, alors les considérations contre manger du chocolat ont plus de poids que celles en faveur de manger du chocolat. Ce que Jean devrait faire, toutes choses bien considérées, est de s'empêcher de manger du chocolat. C'est ce qu'il voudrait le plus faire s'il était pleinement rationnel.

Nous avons donc une autre interprétation de ce que Jean affirme lorsqu'il dit qu'il devrait cesser de manger autant de chocolat. Il exprime la croyance que c'est ce qu'il devrait faire toutes choses bien considérées. Ceci, par ailleurs, est une croyance à propos de ce qu'il voudrait le plus faire s'il était pleinement rationnel simpliciter, c'est-à-dire abstraction faite d'autres exigences, s'il connaissait tous les faits pertinents et s'il était pleinement instrumentalement rationnel. Jean est en position d'avoir cette croyance, parce que, même s'il n'a plus un sens aigu de ce qui constitue une bonne santé, il 
se souvient de ce que c'était lorsqu'il était en bonne santé. Il sait, en d'autre termes, qu'il avait de l'information dans le passé, information qu'il ne possède plus, qui cause en lui le désir très fort d'être en bonne santé. Cependant, parce qu'il n'a plus cette information, il n'a plus un tel désir de fait.

J'ai dit que cette explication de l'histoire de Jean était tout à fait naturelle. Elle est naturelle parce que nous sommes tous familiers avec des situations dans lesquelles, bien que nous soyons pleinement instrumentalement rationnels, nous n'avons aucun désir d'agir de la façon que nous croyons que nous devrions agir si nous étions pleinement rationnels simpliciter. Les trous de mémoire, le manque d'imagination et l'ignorance - et, dans le même registre, l'incohérence, la dépression et tous les autres désordres de personnalité - peuvent causer la perte du désir que nous croyons que nous aurions dans un état plus pleinement rationnel, ou causent en nous d'avoir des désirs que nous croyons que nous aurions dans un état plus pleinement rationnel. Ces types d'échec de la raison sont donc aussi parmi ceux qui peuvent expliquer notre besoin de contrôle de soi, parce qu'ils peuvent aussi expliquer pourquoi nous désirons agir d'une certaine façon, alors que croyons que nous devrions agir d'une autre façon.

\section{Comment un agent peut-il avoir besoin de se contrôler, et réussir à se contrôler ?}

La première énigme consistait à expliquer ce que signifie "doit " lorsque nous disons, comme Jean, que bien que nous puissions désirer agir d'une certaine façon dans certaines circonstances, lorsque nous pensons que nous aurions besoin de nous contrôler dans ces circonstances, ce que nous croyons est aussi que nous ne devrions pas agir de cette manière. La seconde énigme est de faire sens de cette idée que nous devrions nous contrôler dans la mesure du possible.

La source du problème réside dans un truisme en philosophie de l'action, un truisme popularisé par Davidson'2. Selon ce truisme, si un agent désire le plus agir d'une certaine façon, et s'il croit qu'il est libre d'accomplir cette action, alors s'il tente d'accomplir une action, ce sera cette action qu'il tentera d'accomplir. Donc, selon le truisme, si, ici et maintenant, je désire le plus continuer de parler, et si je crois que je puis le faire, alors c'est ce que je tenterai de faire. Bien sûr, je pourrais ne rien faire. Je pourrais m'évanouir, tomber dans le coma ou mourir. Mais si je tente de faire quelque chose, alors ce que je tenterai de faire est de continuer de parler.

2. Davidson, D. "How is Weakness of the Will Possible?", 1970, repris dans Davidson, D. Essays on Actions and Events, Oxford, Oxford University Press, 1980, pp. 21-42 Trad. fr. par P. Engel, "Comment la faiblesse de la volonté est-elle possible ? ", dans D. Davidson, Action et événements, Paris, PUF, 1993, pp. 37-65 
Bien que le truisme requière une formulation plus nuancée que celle que j'ai donnée ici, j'espère que cela a l'air d'un truisme. Il devrait en être ainsi, étant donné la façon dont les concepts de désir, de croyance et d'action sont définis. Une action est définie, après tout, comme quelque chose qui est fait et qui est la conséquence causale d'un désir et d'une croyance et, dans le cas où l'agent aurait une grande variété de désirs et de croyances, ce qu'il veut le plus est simplement défini comme l'action qui est l'objet du désir et de la croyance qui ont le plus grand pouvoir causal. Bien que le truisme requière une formulation plus nuancée, il semble donc légitime de supposer que celuici, ou quelque chose de semblable, est vrai.

Étant donné le truisme, cependant, il est difficile de voir comment quelqu'un qui peut avoir besoin de se contrôler peut réussir à ce faire. Comme nous l'avons vu, un agent qui a besoin de se contrôler désire le plus agir d'une certaine façon alors qu'il croit qu'il devrait agir d'une autre façon. Mais si l'agent désire le plus agir d'une certaine façon alors qu'il croit qu'il devrait agir d'une autre, le truisme nous dit que, s'il tente de faire quelque chose, ce qu'il tentera de faire est d'agir de la façon dont il veut le plus agir, et non de la façon dont il croit qu'il devrait agir. En fait, puisque le truisme se présente comme une vérité conceptuelle, il semble s'ensuivre qu'il soit logiquement impossible pour qui que ce soit qui a besoin de se contrôler de réussir à ce faire, puisque cela exigerait qu'il désire le plus agir d'une certaine façon et désirer encore plus fort agir de la façon exigée par le contrôle de soi. C'est un parfaite contradiction dans la description de l'agent en question.

Considérons le cas de Jean afin d'être clair. Jean désire le plus manger du chocolat, mais croit qu'il ne devrait pas. Suivant le truisme, cependant, il s'ensuit que si Jean essaie de faire quelque chose, il va essayer de manger du chocolat. Mais si Jean va essayer de manger du chocolat, s'il tente de faire quelque chose, alors il ne va bien sûr pas essayer de se contrôler. Bien que nous ayons réussi à expliquer pourquoi Jean a besoin de se contrôler, il semble que ce qu'il a besoin de faire est une impossibilité logique. C'est la seconde énigme concernant le contrôle de soi. Comment la résoudre ?

Il me semble que cette énigme se présente à nous parce que certaines assomptions ont besoin d'être explicitées et questionnées. Notons, par exemple, qu'il y a au moins deux moments du temps différents pendant lesquels il peut se contrôler. Supposons que nous envisageons, au temps t1, que nous serons hors de contrôle au temps $\mathrm{t} 2$ (ou au moins l'absence de contrôle de soi à t2). Les deux moments pendant lesquels nous pouvons nous contrôler reflètent le fait que $\mathrm{t} 1$ et $\mathrm{t} 2$ peuvent être le même moment ou des moments différents. L'énigme surgit seulement si on suppose que $\mathrm{t} 1$ et $\mathrm{t} 2$ sont identiques.

Imaginons que $t 1$ précède $t 2$. Nous sommes donc en mesure de nous demander, à t1, ce que nous voulons le plus faire à $\mathrm{t} 1$. La réponse pourrait bien être, croyant comme nous le faisons qu'à un moment $\mathrm{t} 2$ nous pouvons potentiellement perdre le contrôle, que ce nous voulons le plus faire est de s'assurer de ne pas perdre le contrôle à t2. Et ici, conséquemment, nous 
avons une façon parfaitement directe d'accomplir une action et donc de contrôler nos actions subséquentes, (du moins dans la mesure où nous ne perdons pas le contrôle à t1). Nous pouvons nous contrôler diachroniquement, à un moment qui précède $\mathrm{t} 2$, en organisant les circonstances de l'action que nous affronterons à un moment subséquent de façon à faire disparaître la possibilité de perte de contrôle à ce moment.

Par exemple, si à un moment précédent, alors qu'il était pleinement instrumentalement rationnel, Jean avait prévu qu'il ne serait plus instrumentalement rationnel au moment de passer devant le supermarché en allant au travail - peut être que la vue du supermarché rend particulièrement saillante la possibilité d'acheter du chocolat, ce qui cause le fait qu'il devient instrumentalement irrationnel - alors, s'il avait le plus voulu faire cela, il aurait pu s'assurer d'agir d'une manière instrumentalement rationnelle plus tard, disons en s'assurant que sa seule option aurait alors été celle qu'il aurait désirée s'il avait été pleinement instrumentalement rationnel. Il aurait pu aller au travail en empruntant un chemin différent de façon à ce qu'il ne voit jamais le supermarché ou s'arranger pour ne pas avoir d'argent dans l'auto en allant au travail, ou même emmener quelqu'un avec lui dans l'auto, quelqu'un qui aurait pu lui parler et le convaincre de ne pas s'arrêter pour acheter du chocolat, et ainsi de suite. De cette manière, il aurait pu s'assurer à un moment précédent que, malgré le potentiel d'action instrumentalement irrationnelle à un moment subséquent, au moment de passer devant le supermarché, ce potentiel ne soit jamais réalisé.

En bref, donc, une solution à cette seconde énigme concernant le contrôle de soi se trouve dans l'observation, très simple, que le contrôle de soi diachronique est possible. Lorsque nous nous contrôlons diachroniquement, nos tentatives de ne pas faire ce que nous voulons vraiment faire peut exiger que notre plus fort désir soit de ne pas agir sur la base de notre plus fort désir. Mais il n'y a pas de contradiction, si l'on voit que les désirs sont présents à des moments différents. Lorsque nous nous contrôlons à t1, notre plus fort désir à $\mathrm{t} 1$ peut être ce qui cause que nous n'agissions pas sur la base de ce qui, en l'absence de ce contrôle de soi diachronique, aurait été notre plus fort désir à un moment $\mathrm{t} 2$ subséquent.

\section{Comment un agent peut-il se contrôler, et réussir à ce faire au moment où il est le plus vulnérable ?}

Tout cela peut être parfait. Mais que faire si t 1 et $\mathrm{t} 2$ sont identiques ? Au moment où Jean désire le plus fortement manger du chocolat, Jean est-il capable de ne pas agir sur la base de son désir le plus fort ? C'est notre troisième énigme du contrôle de soi. Étant donné que le contrôle de soi diachronique est possible, le contrôle de soi synchronique est-il aussi possible ? Estil possible pour un agent d'avoir besoin de se contrôler et de réussir à ce faire dans un moment de vulnérabilité ? 
Certains sont sceptiques en ce qui concerne le contrôle de soi synchronique. Une raison de leur scepticisme est que plusieurs exemples de ce qui semble des cas de contrôle de soi synchronique sont en fait, après examen, des cas de contrôle de soi diachronique où les deux moments du temps sont simplement très rapprochés. Ainsi, par exemple, supposons que Jean aperçoit du coin de l'oeil le supermarché où il se rend régulièrement afin d'acheter son chocolat préféré lorsqu'il se rend au travail. Ce coup d'œil causera une modification irrationnelle dans son profil désidératif. Il causera le fait qu'il devient instrumentalement irrationnel, disons, et donc qu'il désire le plus fortement s'arrêter et acheter du chocolat, alors qu'avant le coup d'œil il n'avait pas un tel désir. Si cela est juste, Jean pourrait bien faire quelque chose afin de s'empêcher de jeter un coup d'œil un peu avant de jeter ce coup d'œil. Il pourrait regarder ailleurs, mettre son bras devant ses yeux, porter son attention ailleurs, et quoi encore. Mais bien que, dans de tels cas, il serait naturel de décrire Jean comme ayant repris ses esprits au moment de la vulnérabilité, le fait est que nous imaginons Jean reprendre ses esprits un instant avant qu'il puisse perdre le contrôle. C'est donc un cas de contrôle de soi diachronique, non un cas de contrôle de soi synchronique et, comme tel, ne nous donne aucune contradiction embarrassante dans la description de ce que fait Jean. On peut simplement imaginer que Jean veut le plus s'empêcher de jeter ce coup d'œil au supermarché et agir sur la base de ce désir un moment avant que ses désirs aient changé.

Nous ne pouvons cependant imaginer qu'un agent, au même moment, veut le plus agir d'une certaine façon - la façon requise par la perte de contrôle - et veut le plus agir d'une autre façon — la façon requise par le contrôle de soi. Jean, par exemple, ne peut au même moment vouloir le plus manger du chocolat et vouloir le plus s'empêcher de manger du chocolat, parce que ce serait une contradiction manifeste dans la description de Jean. Les sceptiques à propos du contrôle de soi synchronique mettent, à juste titre, l'emphase sur ce point. La question que nous devons nous poser, néanmoins, est celle de savoir si le contrôle de soi synchronique exige une telle chose.

On pourrait penser qu'il y a une preuve a priori que le contrôle de soi synchronique peut être décrit de façon non contradictoire. La preuve va comme suit. La relation entre les désirs et les actions est causale. Étant donné que les causes précèdent leurs effets, il s'ensuit donc que les désirs causant nos actions sont les désirs que nous avons à un certain moment, et que ce qu'ils causent sont les actions que nous accomplissons un peu plus tard. Puisqu'il en va ainsi, il doit être au moins logiquement possible qu'il y ait un désir plus fort pour causer le contrôle de soi synchronique, même si le contrôle de soi ne fait qu'empêcher l'agent d'agir sur la base d'un désir plus fort qu'il a au moment où il se contrôle.

Afin de voir pourquoi, imaginons que le contrôle de soi prenne place pendant qu'un agent désire le plus agir d'une façon incontrôlée, et imaginons que cela compte comme le contrôle de soi synchronique parce que cela empêche ces désirs d'avoir leurs effets caractéristiques. Puisque le contrôle de soi 
synchronique a été causé par le désir le plus fort que l'agent avait un peu plus tôt, il n'y a tout simplement pas de contradiction dans notre supposition que ces désirs les plus forts, ceux que l'agent avait un peu plus tôt, étaient différents. En d'autres termes, puisque les désirs qui causent un agent à se contrôler synchroniquement sont ceux qu'il a un instant avant de se contrôler synchroniquement, et puisque ce moment, le moment où il se contrôle synchroniquement, est l'instant pendant lequel l'agent désire le plus agir d'une manière incontrôlée, il n'y a tout simplement pas de contradiction impliquée dans notre supposition que ce que l'agent désire le plus faire un instant plus tôt est de se contrôler à l'instant suivant.

Un exemple peut être utile. Supposons que, comme cela semble souvent être le cas, Jean se contrôle en ayant certaines pensées ou en se mettant à rêver. Au moment où il veut le plus manger du chocolat, supposons qu'il pense que le chocolat qu'il s'apprête à manger est un morceau de gras et qu'il imagine le morceau de gras coincé dans son estomac après qu'il l'ait mangé. Cet exercice de l'imagination peut bien empêcher le désir de Jean de manger du chocolat - qui, par stipulation, est le plus fort désir que Jean a en cet instant - d'avoir son effet caractéristique. Mais il n'y a aucune contradiction impliquée dans la supposition qu'imaginer quelque chose était causé par le désir que Jean avait un instant plus tôt, un instant plus tôt pendant lequel ce que Jean voulait le plus faire était imaginer le chocolat comme un morceau de gras coincé dans son estomac, afin d'empêcher le plus fort désir qu'il avait à l'instant d'avoir son effet caractéristique. Le contrôle de soi compte comme synchronique, parce qu'il se produit au moment où Jean désire le plus manger du chocolat. Mais cela ne signifie pas qu'il ne pourrait pas être causé par des désirs plus forts de Jean différents à un instant précédent.

Je dois cependant confesser ne pas être convaincu par cet argument $a$ priori en faveur de contrôle de soi synchronique. Le problème est que le désir qui cause les actions de l'agent ne les initie tout simplement pas. Les actions prennent du temps avant de se produire. Conséquemment, les désirs qui les causent doivent les initier et les soutenir dans les temps. Considérons, par exemple, quelqu'un qui désire le plus lever le bras au-dessus la tête et qui agit sur la base de ce désir, mais qui perd soudainement le désir de lever le bras au-dessus la tête avant que l'acte soit complété. On doit souligner que l'action de lever le bras cesse aussitôt que le désir disparaît. Si le bras est au niveau de ses épaules, il ne continue pas de le monter, parce que cette action était initiée par le désir de lever le bras au-dessus la tête. Son désir de lever le bras au-dessus la tête doit causalement supporter la levée du bras pendant tout le temps pendant lequel elle se produit.

De même, étant donné que cela prend du temps de penser ou de se mettre à imaginer quelque chose dans ce qui constitue une tentative synchronique de contrôle de soi - même une vague pensée prends du temps avant de se produire, comme le fait le flash d'une image devant l'esprit - le désir qui cause la pensée ou le fait d'imaginer quelque chose doit les initier et les 
supporter pendant tout le temps pendant lequel ils se produisent. Un dilemme, pour la thèse voulant que nous avons donné une preuve a priori que le contrôle de soi synchronique peut être décrit en termes non contradictoires, s'offre de lui-même, un dilemme que nous pouvons mettre à l'avant scène en examinant encore une fois le cas de Jean.

Dans la première branche du dilemme, Jean désire le plus avoir une pensée qui l'empêchera d'acquérir un désir plus fort différent dans quelques moments. Par exemple, Jean peut vraiment croire qu'il acquerra le désir de manger du chocolat dans quelques instants, un désir qui sera alors son plus fort désir, s'il ne pense pas le chocolat comme un morceau de gras coincé dans son estomac dans les prochains instants, mais il peut aussi désirer le plus, ici et maintenant, avoir de telles pensées. Dans ce cas, bien que l'on puisse facilement imaginer que le désir de Jean initie et soutient avoir cette pensée pendant les prochains instants, avoir la pensée qu'il a, et donc le contrôle de soi que cela constitue si cela a l'effet désiré, se présente auparavant et ainsi empêche le désir de manger du chocolat. Le contrôle de soi, bien que peut-être une action, est au mieux diachronique.

Dans l'autre branche du dilemme ce que Jean désire le plus faire est ne pas avoir de pensée qui l'empêcherait d'acquérir le désir plus fort dans un moment subséquent, mais d'avoir une pensée qui empêchera le plus fort désir qu'il aura de fait, dans quelques instants, d'avoir ses effets caractéristiques. Par exemple, ici et maintenant, il pourrait vouloir le plus penser, en cet instant, le chocolat comme un morceau de gras coincé dans son estomac parce qu'il croit que, à l'instant suivant, il désirera le plus manger du chocolat, et parce qu'il croit que s'il pense le chocolat comme un vulgaire morceau de gras en cet instant, alors il empêchera son désir d'avoir son effet caractéristique.

Mais si c'est ce que Jean veut le plus faire, alors il est difficile de voir comment son désir peut réellement causer en lui d'avoir une pensée adéquate. Même s'il croit que penser le chocolat comme un vulgaire morceau de gras coincé dans son estomac empêcherait son désir de manger du chocolat d'avoir son effet caractéristique, son présent désir, qui disparaîtra l'instant suivant, pourrait au mieux initier une telle pensée, une pensée qui disparaîtra au moment suivant, alors qu'il n'y a plus aucun désir pour causalement soutenir cette occurrence. Alors Jean, présumément, agira sur la base de son désir le plus fort à l'instant qui suit : son désir de manger du chocolat. Il mangera du chocolat et ainsi échouera à se contrôler synchroniquement. ${ }^{3}$ Il me semble donc que rien de ce qui fut dit jusqu'à maintenant ne nous donne des

3. Cet argument supporte-t-il la conclusion plus forte que le contrôle de soi synchronique que ces actions sont logiquement impossibles, ou simplement la conclusion plus faible que, étant donné les faits empiriques, les pensées qui constituent le contrôle de soi synchronique doivent durer plus longtemps qu'un instant, et donc que nous ne nous contrôlons jamais sous la forme d'une action ? Ceux qui pense que le concept d'une pensée qui dure plus d'un instant est incohérent devrait penser que cela supporte la conclusion la plus forte. Ceux qui pensent que ce concept est cohérent devraient penser qu'elle supporte, au mieux, la conclusion la plus faible. 
raisons de supposer que le contrôle de soi synchronique puisse être décrit en termes non contradictoires

La discussion de cette prétendue preuve a priori que le contrôle de soi synchronique peut être décrit en termes non contradictoires a été utile, parce qu'elle nous a obligés à considérer ce qui me semble des cas beaucoup plus intéressants et éclairants de contrôle de soi apparemment synchroniques. Pensons à une variation sur l'exemple examiné plus haut. Comme il arrive souvent, Jean découvre qu'il veut le plus manger du chocolat. Il en prend, mais, au moment où il s'apprête à le mettre dans sa bouche, il le regarde et commence à imaginer que le chocolat est un morceau de gras qui sera bientôt coincé dans son estomac. C'est, je pense, une expérience commune que d'imaginer de telles choses. Et l'effet causal d'imaginer de telles choses peut être une modification complète et brutale du profil désidératif de l'agent. Bien qu'avant d'imaginer une telle chose Jean désirait le plus manger du chocolat - en fait, il s'apprêtait à en manger - après qu'il ait imaginé un telle chose, il ne veut plus le faire. Il pose le chocolat et fait autre chose.

Ici, me semble-t-il, nous trouvons enfin la question qui a exigé une réponse, dans la mesure où nous supposons que les cas de contrôle de soi synchronique sont possibles. Car, enfin, pourquoi diable supposons-nous que Jean imagine de telles choses ? Le plus fort désir qu'il a à ce moment ne l'a pas causé, c'est sûr. Par stipulation, ce qu'il voulait le plus faire en cet instant est de manger du chocolat, et imaginer une telle chose sape ce désir. Mais le plus fort désir qu'il avait un instant plus tôt ne le cause pas non plus. Encore une fois, par stipulation, ce qu'il voulait le plus faire un instant plus tôt est de manger du chocolat. Mais, dans ce cas, qu'est ce qui cause ce qu'il a imaginé ? La réponse me semble implicite dans ce qui fut dit au tout début de ce texte à propos de ce que "devrait " signifie lorsque Jean dit qu'il ne devrait pas manger autant de chocolat.

Souvenons-nous que Jean a besoin de se contrôler parce bien qu'il croit qu'il ne devrait pas manger autant de chocolat, c'est ce qu'il veut le plus faire. Comme je l'ai déjà soutenu, la croyance de Jean - sa croyance qu'il ne devrait pas manger de chocolat - est simplement la croyance qu'il voudrait le plus ne pas manger de chocolat, dans les circonstances, s'il était pleinement rationnel simpliciter, c'est-à-dire s'il avait l'ensemble de désirs qu'il aurait s'il était pleinement instrumentalement rationnel, si ses désirs était tous à l'abri des effets d'influences distordantes comme la dépression, si ses désirs comme tout formaient un ensemble maximalement éclairé, unifié et cohérent, et ainsi de suite. Le problème de Jean, donc, est que son plus fort désir est en désaccord avec le plus fort désir qu'il croit qu'il aurait dans cet état d'esprit plus pleinement rationnel et cohérent.

Considérons maintenant les deux psychologies suivantes. La première comprend la croyance de l'agent qu'il voudrait le plus, dans les circonstances, agir d'une certaine façon s'il avait un ensemble maximalement cohérent de désirs et, en plus, un désir d'agir de cette façon. L'autre psychologie 
comprend sa croyance qu'il voudrait le plus, dans les circonstances, agir d'une certaine façon s'il avait un ensemble de désirs maximalement cohérent, mais qui ne comprend pas, en plus, le désir d'agir de cette manière. Que peuton dire à propos de ces psychologies à partir de ce que nous avons dit jusqu'à maintenant?

La réponse semble assez claire. On peut dire que la première psychologie montre plus de cohérence que la seconde. Le simple fait que les agents n'arrivent pas à avoir des désirs, concernant quoi faire dans les circonstances actuelles, qu'ils croient qu'ils auraient s'ils avaient un ensemble de désirs maximalement cohérent constitue en soi une sorte d'incohérence ou de déséquilibre dans leur psychologie. Cela constitue une sorte d'incohérence ou de déséquilibre parce que les agents échouent de leur propre point de vue. Bien sûr, ce n'est pas dire que le désir n'est pas cohérent avec d'autres désirs et d'autres croyances de l'agent. Ce pourrait être l'élément crucial dans un ensemble cohérent. Mon point est simplement qu'une source d'incohérence dans la psychologie des agents se situe dans un manque d'harmonie entre leurs désirs concernant ce qu'ils doivent faire dans les circonstances et leurs croyances concernant ce qu'ils voudraient faire, dans les circonstances, s'ils avaient un ensemble maximalement cohérent de désirs.

Le fait qu'il en soit ainsi me semble très significatif. Les agents rationnels possèdent une disposition générale non désidérative sous la forme d'une tendance vers la cohérence. C'est cette tendance qui explique pourquoi, par exemple, leurs croyances tendent à être en conformité avec l'évidence, et que leurs désirs tendent à se développer à la lumière de leurs désirs pour certaines fins et leurs croyances en certains moyens pour atteindre ces fins. Qui plus est, la possession de cette tendance vers la cohérence n'est pas affaire de tout ou rien. Il y a des agents exceptionnellement rationnels pour qui la tendance vers la cohérence semble claire et complète : leurs croyances ne sont jamais en désaccord avec l'évidence, leurs désirs ne sont jamais en désaccord avec leurs désirs pour certaines fins et leur croyance en certains moyens. Et il y a ceux qui, malgré la cohérence d'une bonne partie de leur psychologie, montrent des poches d'incohérence : ils croient parfois contre toute évidence ou n'arrivent pas à désirer les moyens considérés permettre d'atteindre certaines fins. Et il y a ceux qui sont à peine interprétables, étant donné le peu de cohérence montrée par leur psychologie : leurs croyances et leurs désirs constituent un vrai gâchis.

Si j'ai raison, cependant, les agents qui croient qu'ils désireraient agir d'une certaine façon, dans les circonstances, s'ils avaient une psychologie maximalement cohérente, mais n'arrivent pas à avoir le désir correspondant, font preuve de fait d'un manque de cohérence dans leur psychologie, alors il me semble s'ensuivre que cette tendance que possèdent les agents rationnels vers la cohérence peut potentiellement expliquer pourquoi leurs croyances tendent à être en conformité avec l'évidence, et leurs désirs en conformité avec leurs désirs de certaines fins et leurs croyances concernant les moyens 
d'atteindre ces fins, mais aussi pourquoi leurs désirs concernant ce qu'ils feront, dans les circonstances tendent à être en conformité avec leurs croyances concernant ce qu'ils voudraient faire, dans les circonstances, s'ils avaient un ensemble maximalement cohérent de désirs.

Chez les agents qui possèdent cette tendance à un haut degré, par exemple, on peut s'attendre à trouver que leurs croyances concernant ce qu'ils voudraient faire, dans les circonstances, s'ils avaient un ensemble maximalement cohérent de désirs causerait, ou du moins soutiendrait, leurs désirs correspondant d'une façon assez systématique. Toute chose égale par ailleurs, ils n'auraient jamais besoin de se contrôler, parce qu'ils ne se trouveraient jamais dans une situation où ils croient qu'ils désireraient faire quelque chose, dans les circonstances, s'ils avaient un ensemble maximalement cohérent de désirs et ne pas arriver à désirer le faire.

Mais chez les agents qui ne sont pas aussi cohérents, on pourrait s'attendre à ce qu'ils n'aient pas les tendances d'appui, les tendances qui les rendent capable de reprendre la piste lorsque leurs désirs ne sont pas en accords avec leurs croyances concernant ce qu'ils voudraient faire, dans les circonstances, s'ils étaient maximalement cohérents. On pourrait s'attendre à ce qu'ils soient disposés, par exemple, à s'engager dans certains processus de pensée ou de fabulation qui empêchent leurs désirs divergents d'avoir leurs effets caractéristiques, et qui causent le fait qu'ils ont des désirs qui les conduisent à faire ce qu'ils auraient voulu faire s'ils avaient eu un ensemble maximalement cohérent de désirs.

Ici réside l'explication de comment, malgré le fait qu'il ne veuille rien de plus que du chocolat, Jean peut néanmoins imaginer le chocolat comme n'étant rien d'autre qu'un vulgaire morceau de gras coincé dans son estomac. Il peut s'engager dans cet exercice d'imagination, parce qu'il possède une tendance, assez générale, vers la cohérence dans sa psychologie, et cette tendance cause le fait qu'il pense de telle chose et s'engage dans de tels exercices d'imagination afin de conférer à sa psychologie un état plus cohérent étant donné le présent état d'incohérence. En d'autres termes, Jean imagine que le chocolat est un morceau de gras coincé dans son estomac parce qu'il est rationnel, et que c'est la chose qu'il est rationnel pour lui d'imaginer en cet instant. C'est la chose rationnelle pour lui à imaginer parce que cela cause le fait qu'il perd le désir de manger du chocolat - le désir dont la présence rend sa psychologie incohérente - et, en lieu et place, cause le fait de désirer le plus faire ce qu'il croit qu'il voudrait le plus faire s'il avait un ensemble maximalement éclairé, cohérent et unifié de désirs. De cette manière, Jean restaure la cohérence dans sa psychologie.

La troisième énigme concernant le contrôle de soi surgit à cause de l'apparente impossibilité logique du contrôle de soi synchronique, c'est-àdire de cas dans lesquels les agents ont besoin à la fois de se contrôler et de réussir à le faire dans un moment de vulnérabilité. Ces cas paraissent impossibles parce que leur description semble impliquer une contradiction. Il 
semble que les agents doivent à la fois vouloir le plus agir d'une façon incontrôlée, et vouloir le plus agir d'une façon requise par le contrôle de soi. Mais la solution à cette énigme réside dans le fait que ce qui rend le contrôle de soi synchronique possible est le fait que ce n'est pas du tout une action - ou, en tout cas, ce n'est pas une action dans le sens ordinaire du mot « action » saisi dans le truisme formulé plus haut, le sens d' " action » dans lequel les actions sont des choses que nous faisons et qui sont causées par des désirs et des croyances concernant la relation moyen/fin. Lorsque les agents se contrôlent synchroniquement, ils s'engagent dans divers processus et imaginent des choses, et faire ainsi est causé par une tendance non désidérative qu'ils possèdent, la tendance de leur psychologie à tendre vers la cohérence, et non par des désirs ou des croyances concernant la relation moyen/fin qu'ils peuvent posséder.

\section{Comment le contrôle de soi peut-il être autre chose qu'une action ?}

Ceci nous amène à la quatrième et dernière énigme du contrôle de soi. Comment le contrôle de soi peut-il être autre chose qu'une action ? Le contrôle de soi est, après tout, quelque chose que nous faisons - c'est une activité - et les activités sont sûrement des actions.

Mais cette tendance à penser que le contrôle de soi est une action est, je crois, une généralisation hâtive bien compréhensible. Nous commencons par noter que le contrôle de soi est quelque chose que nous faisons. Ensuite nous généralisons : puisque toutes les choses que nous faisons sont des activités, et toutes les activités des actions, nous concluons que le contrôle de soi est une action. Mais c'est une généralisation hâtive parce que tout ce que nous faisons, et toutes nos activités, ne constituent pas des actions. Penser est quelque chose que, normalement, nous disons faire : c'est une activité. Mais ce n'est certainement pas une action en un sens ordinaire de ce terme. Inférer une conclusion de prémisses que nous acceptons est aussi quelque chose que nous faisons - c'est aussi une activité - mais ce n'est pas une action, en aucun sens de ce terme. Dériver un désir, comme moyen, à partir d'un désir pour une fin et une croyance que ce moyen est un moyen pour atteindre cette fin est aussi quelque chose que nous faisons, de même que se souvenir, etc. Mais rien de tout cela ne peut être conçu comme une action - pas, en tout cas, en un sens ordinaire du terme " action ", le sens dans lequel les actions sont des choses que nous faisons et qui sont causées par des désirs et des croyance concernant la relation moyen/fin.

Je suis donc tout à fait heureux de penser qu'il est faux que le contrôle de soi constitue une action. En fait, j'accueille cette conclusion à bras ouverts, parce que le contrôle de soi n'est pas une action. Le contrôle de soi est une pure cognition, et si des actions sont les conséquences du contrôle de soi, alors nous avons des cas où le contrôle de soi nous donne une illustration 
dramatique de la façon dont les actions peuvent être le produit de pures cognitions. Qui plus est, comme nous l'avons vu, l'illustration elle-même est parfaitement ordinaire. Elle ne requiert rien de bizarre de notre part dans le pouvoir de la rationalité. Je vais l'expliquer une dernière fois.

Les agents rationnels possèdent la capacité de réfléchir à leurs propres actions et de se demander ce qu'ils voudraient faire, dans les circonstances, s'ils avaient une psychologie maximalement éclairée, unifiée et cohérente. Ils possèdent aussi diverses tendances qui leur permettent de restaurer la cohérence dans leur psychologie globale. Dans le cas de Jean, parce qu'il croit qu'il désirerait ne pas manger de chocolat, dans les circonstances, s'il avait une psychologie maximalement éclairée, unifiée et cohérente, mais désire le plus manger du chocolat, sa tendance le cause à imaginer que le chocolat est un morceau de gras coincé dans son estomac, et ce qu'il imagine cause en retour un changement crucial dans ses désirs. Il en vient à désirer le plus d'agir d'une façon qu'il croit qu'il voudrait agir, dans les circonstances, s'il avait une psychologie maximalement éclairée, unifiée et cohérente. Lorsqu'il agit, c'est ce qu'il fait.

Malgré le rôle constitutif jouée par les désirs et les croyances concernant la relation moyen/fin de Jean dans la production de ses actions, il arrive que la racine causale de ses actions ne puisse être que sa capacité de réflexion rationnelle - la capacité qui le rend capable de former des croyances quant à ce qu'il voudrait faire dans les circonstances s'il avait une psychologie maximalement éclairée, unifiée et cohérente - d'une part, et la tendance de sa psychologie vers une cohérence totale - la tendance qui le rend capable de s'engager dans l'exercice de l'imagination qui à un effet crucial sur ses désirs d'autre part. Tout le reste découle causalement à partir de là. Si nous avons besoin d'une illustration de la pure raison pratique en action, je ne pense pas que nous puissions trouver un meilleur exemple. ${ }^{4}$

4. Cet article, ici traduit de l'anglais par Richard Vallée, reprend tout en les développant des thèses émises dans plusieurs articles déjà publiés. Ces articles incluent Kennett, J. et M. Smith, "Frog and Toad Lose Control ", Analysis, 1996, pp. 63-73, et "Synchronic SelfControl is Always Non-Actional ", Analysis, 1997, pp. 121-131, (une réponse à Mele, «Underestimating Self-Control »: Kennett and Smith, « On Frog and Toad », Analysis, 1997, pp. 119-123.) ; Pettit P. et M. Smith "Practical Unreason », Mind, 1993a, pp. 53-79, et "Brandt on Self-Control » dans Brad Hooker (dir. de publication) Rationality, Rules and Utility, 1993b, Westview Press, pp. 33-50, 1996, Smith, M., "Valuing : Desiring or Believing ?", dans Charles, David et Kathleen Lennon (dir. de publication) Reduction, Explanation, Realism, Oxford, Oxford University Press, 1992, pp. 323-360, The Moral Problem, Oxford, Basil Blackwell, 1994, "Internal Reasons ", Philosophy and Phenomenological Research, 1995, pp. 109-131 et "A Theory of Freedom and Responsibility ", dans Cullity, Garrett et Berys Gaut (dir. de publication) Ethics and Practical Reason, Oxford, Oxford University Press, 1997, pp. 293-319. 